\title{
Plexiform fibromyxoma with cotyledon-like serosal growth: A case report of a rare gastric tumor and review of the literature
}

\author{
JOSHUA ROBERT KANE ${ }^{1}$, NATASHA LEWIS ${ }^{1}$, REBECCA LIN $^{1}$, CELINA VILLA $^{1}$, ALEXANDRA LARSON $^{1}$, \\ JEFFREY D. WAYNE ${ }^{2}$, ANJANA V. YELDANDI ${ }^{1}$ and WILLIAM B. LASKIN ${ }^{1}$
}

Departments of ${ }^{1}$ Pathology and ${ }^{2}$ Surgery, Feinberg School of Medicine, Northwestern University, Chicago, IL 60611, USA

Received December 29, 2014; Accepted September 29, 2015

DOI: $10.3892 / 01.2016 .4185$

\begin{abstract}
Plexiform fibromyxoma is a rare, benign mesenchymal neoplasm that predilects the gastric antrum and has potential for misdiagnosis as a gastrointestinal stromal tumor (GIST). The histology of the tumor is characterized by interwoven fascicular growth of cytologically bland spindled cells within a variably myxoid stroma. The current study reports the clinicopathological and immunohistochemical findings of a plexiform fibromyxoma resected from a 28 -year-old Vietnamese female. The patient presented with acute, severe abdominal pain and worsening anemia. The initial fine-needle aspiration and needle core biopsy of the gastric antral mass led to an initial diagnosis of GIST. The subsequent distal partial gastrectomy revealed a $5.5-\mathrm{cm}$ transmural antral mass that ulcerated the overlying mucosa and grew as variably elongated, myxoedematous, polypoid (cotyledon-like) excrescences from the serosal surface. Microscopically, the tumor demonstrated plexiform and multinodular growth of cytologically bland spindled cells proliferating in an abundant myxocollagenous stroma with a prominent capillary network. Tumor cells immunohistochemically expressed smooth muscle actin and CD10, but did not express CD117, Discovered on GIST-1 or nuclear $\beta$-catenin. Follow-up evaluation 23 months post surgery revealed no evidence of residual tumor. A review the cases of this rare entity reported in the English language literature is also provided.
\end{abstract}

\section{Introduction}

Plexiform fibromyxoma is a one of the very few types of mesenchymal neoplasm that predilect the stomach. Miettinen et al (1) published the largest series on the topic in 2009, coining the term 'plexiform fibromyxoma' for this benign

Correspondence to: Dr William B. Laskin, Department of Pathology, Feinberg School of Medicine, Northwestern University, 251 East Huron Street, Chicago, IL 60611, USA

E-mail:wb1769@northwestern.edu

Key words: plexiform fibromyxoma, gastrointestinal stromal tumor, immunohistochemistry, stomach, cotyledon-like growth spindle cell tumor. Although alternative designations for the process, including 'plexiform angiomyxoid myofibroblastic tumor' (2-9) and 'plexiform angiomyxoid tumor' (10), have been proposed, these appellations fail to adequately convey the benign nature of the lesion. Indeed, plexiform fibromyxoma is the term currently accepted by the World Health Organization for this tumor (11).

Microscopically, the tumor exhibits a characteristic plexiform growth pattern of small spindled cells in a myxoid or fibromyxoid stroma. The tumor shares a myofibroblastic immunophenotype with gastrointestinal stromal tumors (GISTs), which are the most common mesenchymal tumor of the stomach with an estimated incidence of $>150$ cases for every 1 case of plexiform fibromyxoma; although immunohistochemical positivity of CD117 and Discovered on GIST-1 (DOG-1) in GISTs aids in distinguishing the two entities (1). This distinction is important as GISTs possess a greater potential for aggressive pathobiological behavior, whereas plexiform fibromyxomas are considered to be largely benign.

The present study reports the clinicopathological features of a plexiform fibromyxoma, excised from a 28 -year-old female, that grew as multiple, variably sized, bulbous myxoedematous polypoid projections from the serosal surface. In supplement, a review of the pertinent English language literature regarding this rare neoplasm is presented.

\section{Case report}

A 28-year-old Vietnamese female presented to Northwestern Memorial Hospital (Chicago, IL, USA) in June 2013 with acute, severe abdominal pain and worsening anemia. Computed tomography (CT) of the abdomen and pelvis revealed a lobulated, heterogeneously-enhancing mass with both a solid and cystic component in the wall of the gastric antrum (Fig. 1A). A fine-needle aspiration and needle core biopsy of the gastric mass demonstrated a myxoid spindle cell lesion with features suggestive of a GIST. As a result of these findings, the patient underwent a distal gastrectomy with a Roux-en-Y gastrojejunostomy.

The distal gastrectomy specimen contained a $5.5 \times 3.5-\mathrm{cm}$, stellate-shaped, multinodular, yellow-tan-colored mass centered in the antral wall and which ulcerated the overlying mucosa (Fig. 1B) and extended onto the serosal surface as multiple, variably sized, myxoedematous polyps imparting 

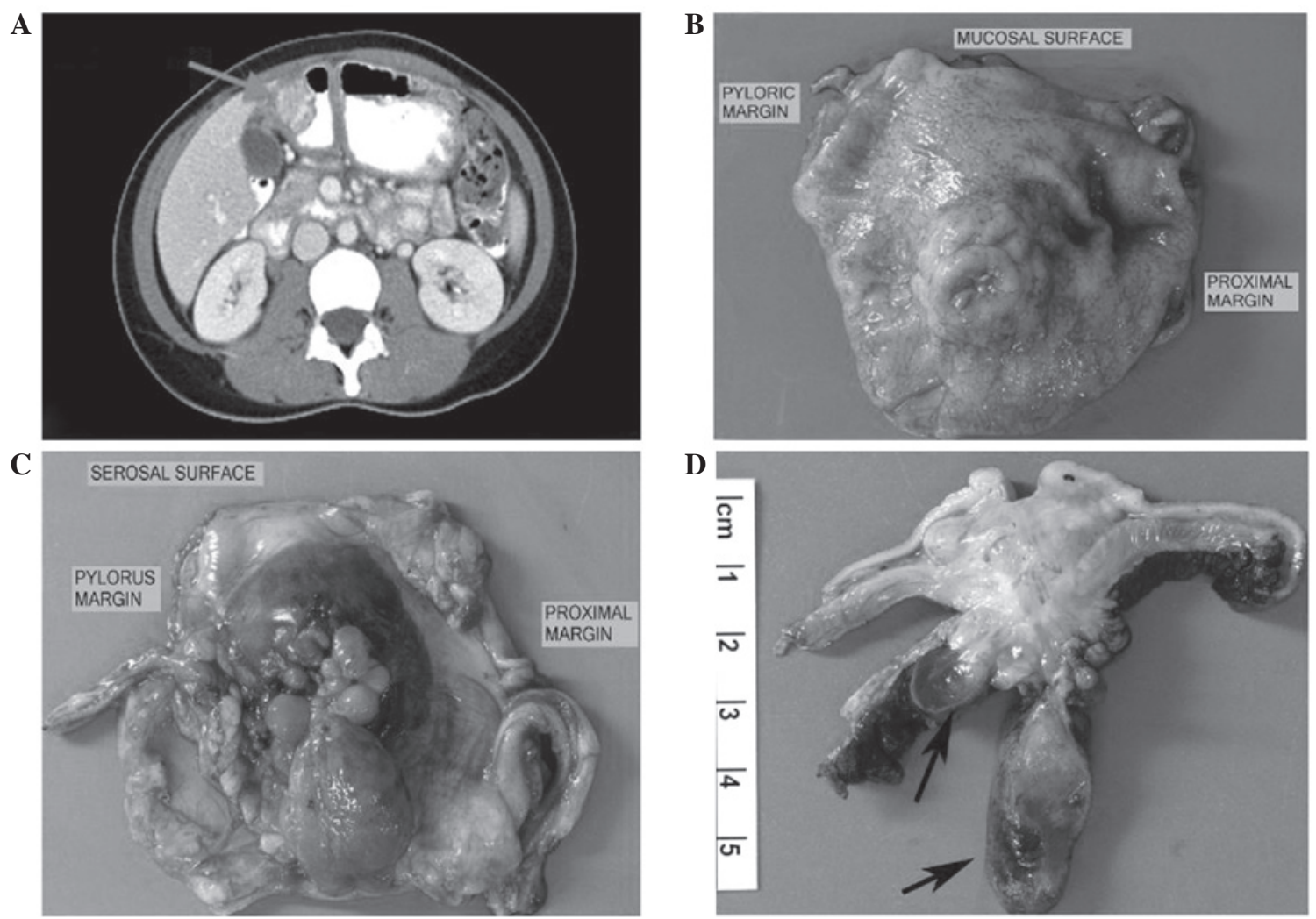

Figure 1. (A) Computed tomography scan of abdomen and pelvis demonstrating a lobulated, heterogeneously-enhancing mass with both solid and cystic components in the gastric antrum (arrow). (B-D) Gross features of plexiform fibromyxoma (distal gastrectomy specimen): (B) Mucosal surface overlying the 5.5-cm tumor is elevated and focally ulcerated; (C) the serosa of the antrum exhibits multiple, variably sized, myxoedematous polypoid (cotelydon-like) structures; (D) the cut surface of the stomach reveals an ill-defined, white-tan-colored, multinodular mass involving the entire stomach wall and ulcerating the overlying mucosa, with multiple, variably sized, polypoid tumor excrescences extending from the serosal surface (arrows).

a cotelyedon-like appearance to the serosal aspect of the stomach (Fig. 1C and D).

At low-power microscopic magnification, intertwined pale, myxocollagenous nodules and fascicles of tumor infiltrated the entire thickness of the gastric wall (Fig. 2A and B) and formed highly myxoedematous polypoid structures on the serosal surface. The myxocollagenous fascicles and nodules of tumor possessed an accentuated vascular component composed of non-branching capillaries and scattered mast cells (Fig. 2C). High-power microscopic evaluation revealed mildly cellular fascicles of cytologically bland spindled cells with scanty, pale, eosinophilic cytoplasm and indistinct cell borders (Fig. 2D). The mitotic count was 7 mitoses per 50 high-power fields, with no atypical mitotic figures identified. No necrosis was observed within the lesional tissue. All margins of resection were free of tumor. The intact mucosa overlying the tumor exhibited superficial chronic inflammation, but no Helicobacter pylori organisms were visually identified.

Immunohistochemically, the spindle cells diffusely expressed smooth muscle actin (SMA) (Fig. 3A) and focally expressed CD10 (Fig. 3B), but were negative for CD117, DOG-1, CD34, S-100 protein, D2-40, keratin AE1/3 and nuclear $\beta$-catenin.

A follow-up CT scan performed one month after the surgery revealed no intra-abdominal pathology, and an esophagogastroduodenoscopy performed due to symptoms of dyspepsia 23 months following surgery revealed moderate stenosis of the gastrojejunal anastomosis, but no evidence of residual or recurring tumor. Any future follow-up for this benign tumor is presently on an 'as needed' basis.

\section{Discussion}

Plexiform fibromyxoma is a rare mesenchymal neoplasm that predilects the stomach and, as demonstrated in the present case, has the potential for misdiagnosis as the more common and often aggressive GIST.

In the English language literature, 32 previously reported cases of plexiform fibromyxoma were identified (Table I) (1-10,12-18). The tumor has been documented in 19 females and 13 males, ranging in age from 7 to 75 years (mean, 40 years; median, 43 years). Symptoms reported at presentation vary and are non-specific, but most commonly include abdominal pain, ulceration, anemia and mass effects. The antrum of the stomach is involved in $88 \%$ of reported cases, with contiguous involvement of the pylorus and/or duodenum in an additional 7 cases. Isolated examples of plexiform fibromyxoma have been documented in the gastric fundus (14), cecum (13), pyloroduodenal region (16) and posterior mediastinum (16).

Grossly, the neoplasm ranges in size from 1.5 to $15 \mathrm{~cm}$ (median, $4.3 \mathrm{~cm}$; mean, $5.2 \mathrm{~cm}$ ). The cut surface of the lesion reveals a multinodular mass centered in the muscularis propria, with dome-shaped elevation and often ulceration of the mucosal surface. Subserosal nodules $(1,2)$ and polypoid projections (10) have been briefly mentioned in a few reports 
A

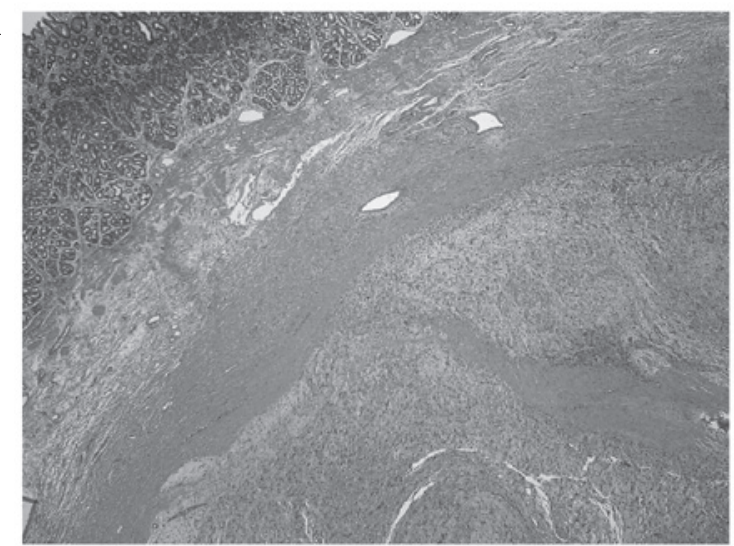

C

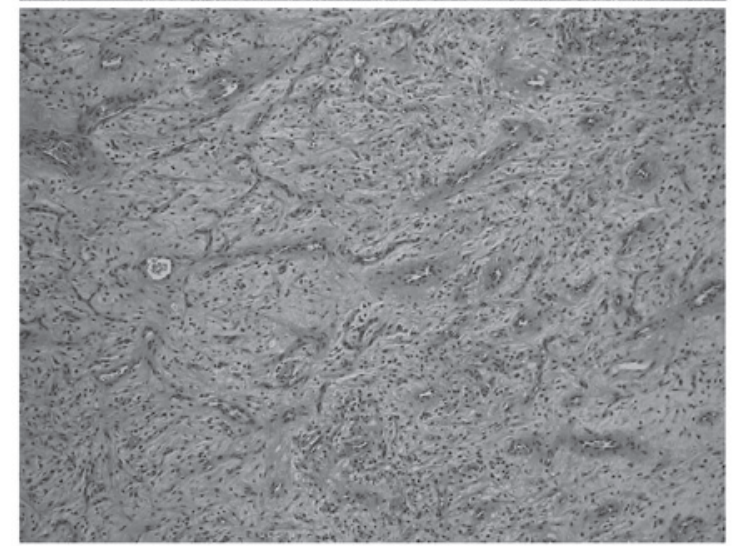

B
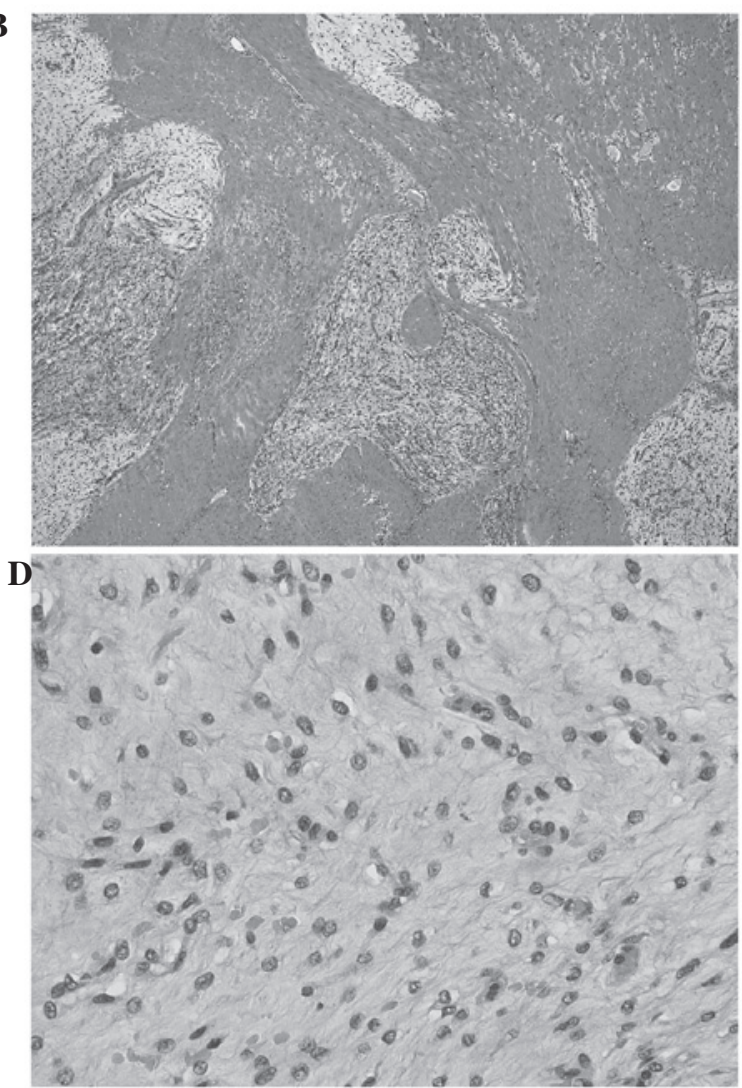

Figure 2. Microscopic evaluation of plexiform fibromyxoma. (A and B) Low-power magnification (4x; hematoxylin and eosin) of plexiform fibromyxoma reveals pale, myxoid fascicles and nodules of tumor infiltrating the muscularis propria of the stomach. (C) Spindled tumor cells proliferating in a myxoid stroma with numerous small, thin-walled vessels (10x magnification; hematoxylin and eosin). (D) High-power magnification shows scattered short, spindled cells with scanty, eosinophilic cytoplasm and oval nuclei harboring evenly dispersed chromatin and inconspicuous nucleoli (20x magnification; hematoxylin and eosin).
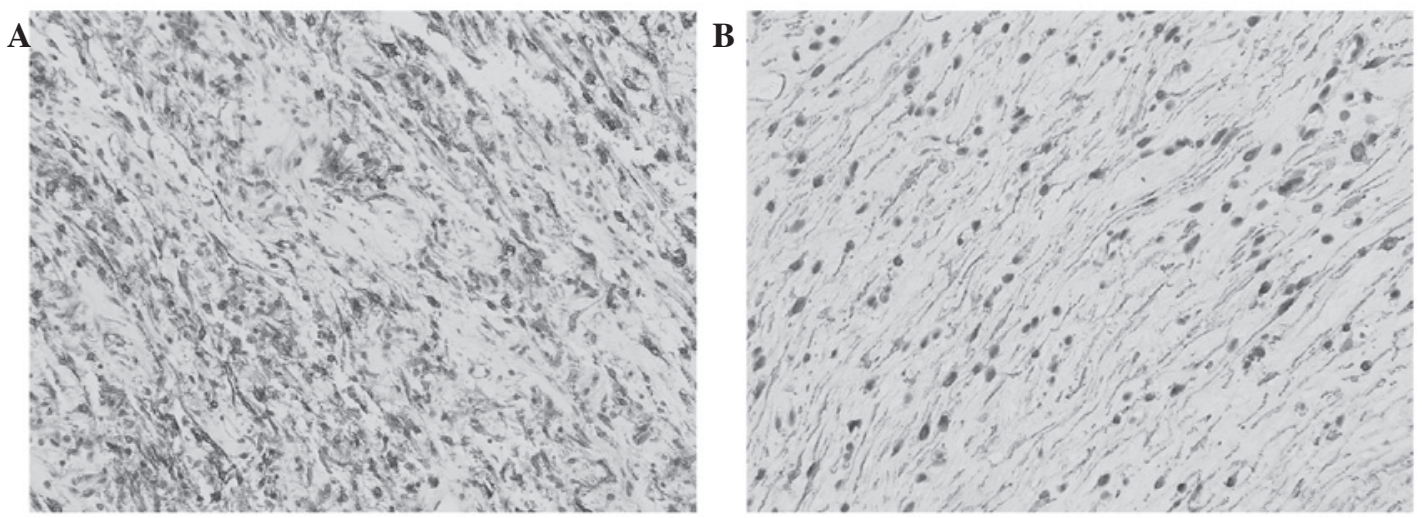

Figure 3. Immunohistochemical profile of plexiform fibromyxoma includes (A) smooth muscle actin and (B) CD10 expression (20x magnification).

detailing the gross appearance of plexiform fibromyxoma. Although GISTs may present primarily as an extramural polypoid mass in the stomach (19), the presence of multiple subserosal myxoedematous polyps, as in the current case, is not a macroscopic feature of GISTs, whereas as it is in plexiform fibromyxoma and therefore may be used to differentiate the two tumor entities on a macroscopic level.

We contend that the light microscopic features of plexiform fibromyxoma are relatively unique and allow an unequivocal diagnosis in the majority of cases. The process is characterized by an interwoven growth pattern of pale-appearing, mildly cellular fascicles and nodules of cytologically bland, short spindled cells proliferating in the myxoid and/or myxocollagenous stroma with an enriched network of simple capillary-sized vessels.

Immunohistochemically, plexiform fibromyxoma demonstrates a myofibroblastic phenotype with documented expression of SMA ( $\mathrm{n}=26$ cases) $(1,2,4-10,14-18)$, vimentin $(n=8)(2,4,6,13,14,16,18)$, muscle specific actin $(n=6)(5,6,10,15)$, desmin $(n=9)(4,5,7,8,10,16-18)$, caldesmon $(n=5)(5,6,10,18)$ and calponin $(n=3)(5,10,16)$. Immunoexpression of CD10 $(n=2)(1)$, nestin $(n=1)(16)$ and progesterone receptor protein $(n=1)(5)$ have also been documented. In the majority of reports $(3,6,7,9,10,14,17,18), \mathrm{Ki}-67$ immunoexpression is 


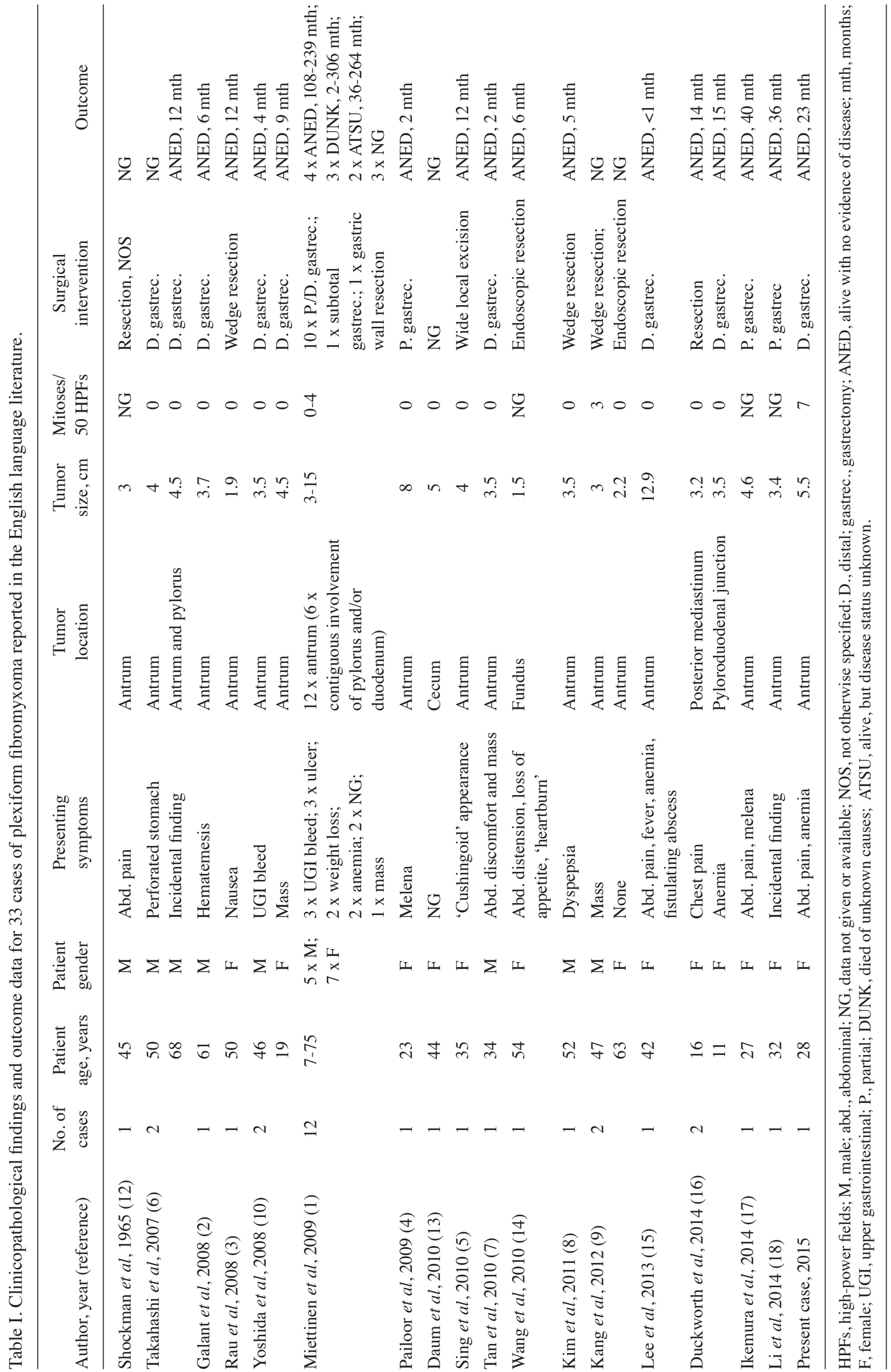


generally very low $(\leq 2 \%)$. Notably, tumor cells do not express CD117 (cKIT), CD34, DOG-1, neurofilament, S-100 protein, nuclear $\beta$-catenin, epithelial membrane antigen, activin receptor-like kinase 1 (ALK-1), CDK4, Muc-4, estrogen receptor protein or keratins.

Mutations in key exons ('hotspots') of the CD117 and platelet-derived growth factor receptor- $\alpha$ (PDGF $\alpha)$ genes are important in the pathogenesis and treatment of the GIST, and are found in $\sim 85 \%$ of cases (20). Molecular evaluation of GIST mutational 'hotspots' in these two genes has revealed 'wild-type' sequences in all cases of plexiform fibromyxoma tested $(1,7,9,10,13)$.

Plexiform fibromyxoma acts in a benign fashion with surgical excision despite the occasional presence of extragastric extension and/or lymphatic/vascular-space invasion by the tumor (1). To date, 21 patients have been reported to be alive without recurrent/persistent disease during follow-up periods ranging from 1 to 239 months (mean, 48 months; median, 12 months) (1-10,12-18).

The differential diagnosis of plexiform fibromyxoma includes other myxoid spindle cell processes that involve the stomach. The myxoid variant of GIST is the predominant entity to consider in the differential diagnosis. Apart from plexiform epithelioid cell GIST variants (21), conventional GISTs typically form a solitary nodular or, in certain cases, a dumbbell-shaped mass. Microscopically, GISTs are composed of larger spindled cells with more abundant, lightly eosinophilic cytoplasm that are arranged in more compact and cellular fascicles. An exaggerated degree of nuclear pallisading and/or perinuclear vacuolization is identified in certain spindle cell variants. Despite immunoexpression of muscle actins in both entities, GISTs typically express CD117 and DOG-1 (>90\% of cases) (22) and CD34 (>80\% of cases) (23).

The inflammatory fibroid polyp is a benign lesion that has a predilection for the gastric antrum (24). The process arises from the submucosa and may also involve the muscularis propria. In contrast to plexiform fibromyxoma, the lesion grows as a solitary and, often, polypoid mass. It is composed of cytologically bland epithelioid and short spindled cells typically arranged concentrically around vessels in a loose fibromyxoid stroma. The accompanying inflammatory infiltrate, rich in eosinophils, is a hallmark of the process and further helps to distinguish the lesion from fibromyxoma. Immunohistochemically, the inflammatory fibroid polyp is positive for CD34, but not SMA (20). PDGFa gene mutations are common in the lesion (25).

Gastrointestinal schwannoma is a benign peripheral nerve sheath tumor that arises primarily as an intramural gastric or colonic mass $(26,27)$. Unlike plexiform fibromyxoma, gastrointestinal schwannoma grows as a solitary, unencapsulated nodule and frequently exhibits lymphoid aggregates or follicles at its periphery. The cells of schwannoma are typically larger than those of fibromyxoma and exhibit characteristic nuclear contour irregularities. Gastrointestinal schwannomas demonstrate strong and diffuse immunoexpression of S-100 protein, and little to no expression of SMA.

The myxoid variant of leiomyoma, on occasion, may exhibit plexiform growth. In contrast to fibromyxoma, the cells of leiomyoma are typically larger with centrally located, blunt-ended nuclei and brightly eosinophilic fibrillary cytoplasm, and frequently grow in a packeted or trabecular pattern. The immunoprofiles of the two lesions include similar expression of myoid markers.

Intra-abdominal fibromatosis is a locally aggressive myofibroblastic neoplasm that may arise from the pelvis or abdominal soft tissue, and can secondarily invade the walls of gastrointestinal viscera, thereby mimicking a primary process. It has a distinctly infiltrative growth pattern and does not exhibit true plexiform architecture. Although myxoid change may occur in fibromatosis, the lesion differs from fibromyxoma by its possession of larger cells that are evenly distributed within distinct, elongated fascicles. Along with SMA immunoexpression, fibromatosis characteristically exhibits nuclear expression of $\beta$-catenin in $75-90 \%$ of cases $(28,29)$.

Mesenteric inflammatory myofibroblastic tumor has the potential to involve the gastric wall, simulating a primary lesion. The lesion may exhibit myxoid areas with spindled tumor cells growing in a fasciitis-like pattern. This process differs from fibromyxoma by exhibiting multi-patterned growth, a distinct population of larger cells with eosinophilic cytoplasm, and a conspicuous lymphoplasmacytic infiltrate (30). In addition to SMA, $>40 \%$ of cases exhibit ALK-1 immunoexpression (31).

In summary, the current study reports a case of plexiform fibromyxoma, a rare mesenchymal gastric neoplasm that requires distinction from the more aggressive GIST. Attention to the characteristic gross and microscopic growth patterns, as well as the absence of CD117 and DOG-1 immunoexpression, may aid in separating these two entities. We also contend that the macroscopic presence of multiple, myxoedematous, serosal polyps should strongly suggest a diagnosis of plexiform fibromyxoma as opposed to GIST or myoid spindle cell lesions.

\section{References}

1. Miettinen M, Makhlouf HR, Sobin LH and Lasota J: Plexiform fibromyxoma: A distinctive benign gastric antral neoplasm not to be confused with a myxoid GIST. Am J Surg Pathol 33: $1624-1632,2009$

2. Galant C, Rousseau E, Ho Minh Duc DK and Pauwels P: Re: Plexiform angiomyxoid myofibroblastic tumor of the stomach. Am J Surg Pathol 32: 1910; author reply 1912-1913, 2008.

3. Rau TT, Hartmann A, Dietmaier W, Schmitz J, Hohenberger W, Hofstaedter F and Katenkamp K: Plexiform angiomyxoid myofibroblastic tumour: Differential diagnosis of gastrointestinal stromal tumour in the stomach. J Clin Pathol 61: 1136-1137, 2008.

4. Pailoor J, Mun KS, Chen CT and Pillay B: Plexiform angiomyxoid myofibroblastic tumour of the stomach. Pathology 41: 698-699, 2009.

5. Sing Y, Subrayan S, Mqadi B, Ramdial PK, Reddy J, Moodley MS and Bux S: Gastric plexiform angiomyxoid myofibroblastic tumor. Pathol Int 60: 621-625, 2010.

6. Takahashi Y, Suzuki M and Fukusato T: Plexiform angiomyxoid myofibroblastic tumor of the stomach. World J Gastroenterol 16: 2835-2840, 2010.

7. Tan CYS, Santos LD and Biankin A: Plexiform angiomyxoid myofibroblastic tumour of the stomach: A case report. Pathology 42: 581-583, 2010.

8. Kim A, Bae YK, Shin HC and Choi JH: Plexiform angiomyxoid myofibroblastic tumor of the stomach: A case report. J Korean Med Sci 26: 1508-1511, 2011.

9. Kang Y, Jung W, Do IG, Lee EJ, Lee MH, Kim KM and Choi J: Plexiform angiomyxoid myofibroblastic tumor of the stomach: Report of two cases and review of the literature. Korean J Pathol 46: 292-296, 2012.

10. Yoshida A, Klimstra DS and Antonescu CR: Plexiform angiomyxoid tumor of the stomach. Am J Surg Pathol 32: 1910-1912; author reply 1912-1913, 2008. 
11. Miettinen M,Blay JY, Kindblom LG and Sobin LH: Mesenchymal tumours of the colon and rectum. In: World Health Organization Classification of Tumours - Pathology and Genetics of Tumours of the Digestive System. Hamilton SR and Aaltonen LA (eds). IARC Press, Lyon, France, pp142, 2000.

12. Shockman AT and Rosen JH: Fibromyxoma of the stomach. Del Med J 37: 225-228, 1965.

13. Daum O, Jirasek T, Grossmann P, Mukensnabl P and Michal M: Plexiform fibroma of the colon. Appl Immunohistochem Mol Morphol 18: 483-484, 2010.

14. Wang WY, Li JN and Li GD: Plexiform angiomyxoid myofibroblastic tumour of the gastric fundus: Successful diagnosis and treatment by endoscopy. J Clin Pathol 63: 569-570, 2010.

15. Lee PW, Yau DT, Lau PP and Chan JK: Plexiform fibromyxoma (plexiform angiomyxoid myofibroblastic tumor) of stomach: An unusual presentation as a fistulating abscess. Int J Surg Pathol 22: 286-290, 2014

16. Duckworth LV, Gonzalez RS, Martelli M, Liu C, Coffin CM and Reith JD: Plexiform fibromyxoma: Report of two pediatric cases and review of the literature. Pediatr Dev Pathol 17: 21-27, 2014.

17. Ikemura M, Maeda E, Hatao F, Aikou S, Seto Y and Fukayama M Plexiform angiomyxoid myofibroblastic tumor (PAMT) of the stomach. A case report focusing on its characteristic growth pattern. Int J Clin Exp Pathol 7: 685-689, 2014.

18. Li P, Yang S, Wang C, Li Y and Geng M: Presence of smooth muscle cell differentiation in plexiform angiomyxoid myofibroblastic tumor of the stomach: A case report. Int J Clin Exp Pathol 7: 823-827, 2014

19. Agaimy A and Wunsch PH: Gastrointestinal stromal tumours: A regular origin in the muscularis propria, but an extremely diverse gross presentation. A review of 200 cases to critically re-evaluate the concept of so-called extra-gastrointestinal stromal tumours. Langenbecks Arch Surg 391: 322-329, 2006.

20. Miettinen $\mathrm{M}$ and Lasota J: Gastrointestinal stromal tumors: Pathology and prognosis at different sites. Semin Diagn Pathol 23: 70-83, 2006.

21. Miettinen M, Wang ZF, Sarlomo-Rikala M, Osuch C, Rutkowski P and Lasota J: Succinate dehydrogenase-deficient GISTs: A clinicopathologic, immunohistochemical, and molecular genetic study of 66 gastric GISTs with predilection to young age. Am J Surg Pathol 35: 1712-1721, 2011.
22. Miettinen M, Sobin LH and Lasota J: Gastrointestinal stromal tumors presenting as omental masses-a clinicopathologic analysis of 95 cases. Am J Surg Pathol 33: 1267-1275, 2009.

23. Miettinen $M$, Sobin LH and Sarlomo-Rikala M Immunohistochemical spectrum of GISTs at different sites and their differential diagnosis with a reference to CD117 (KIT). Mod Pathol 13: 1134-1142, 2000.

24. Liu TC, Lin MT, Montgomery EA and Singhi AD: Inflammatory fibroid polyps of the gastrointestinal tract: Spectrum of clinical, morphologic and immunohistochemistry features. Am J Surg Pathol 37: 586-592, 2013.

25. Lasota J, Wang ZF, Sobin LH and Miettinen M: Gain-of-function PDGFRA mutations, earlier reported in gastrointestinal stromal tumors, are common in small intestinal inflammatory fibroid polyps. A study of 60 cases. Mod Pathol 22: 1049-1056, 2009.

26. Daimaru Y, Kido H, Hashimoto H and Enjoji M: Benign schwannoma of the gastrointestinal tract: A clinicopathologic and immunohistochemical study. Hum Pathol 19: 257-264, 1988

27. Miettinen M, Shekitka KM and Sobin LH: Schwannomas in the colon and rectum: A clinicopathologic and immunohistochemical study of 20 cases. Am J Surg Pathol 25: 846-855, 2001

28. Bhattacharya B, Dilworth HP, Iacobuzio-Donahue C, Ricci F, Weber K, Furlong MA, Fisher C and Montgomery E: Nuclear beta-catenin expression distinguishes deep fibromatosis from other benign and malignant fibroblastic and myofibroblastic lesions. Am J Surg Pathol 29: 653-659, 2005.

29. Montgomery E, Torbenson MS, Kaushal M, Fisher C and Abraham SC: Beta-catenin immunohistochemistry separates mesenteric fibromatosis from gastrointestinal stromal tumor and sclerosing mesenteritis. Am J Surg Pathol 26: 1296-1301, 2002.

30. Coffin CM, Dehner LP and Meis-Kindblom JM: Inflammatory myofibroblastic tumor, inflammatory fibrosarcoma and related lesions: An historical review with differential diagnostic considerations. Semin Diagn Pathol 15: 102-110, 1998.

31. Cessna MH, Zhou H, Sanger WG, Perkins SL, Tripp S, Pickering D, Daines C and Coffin CM: Expression of ALK1 and p80 in inflammatory myofibroblastic tumor and its mesenchymal mimics: A study of 135 cases. Mod Pathol 15: 931-938, 2002. 